recent example, published in September (N. Engl. J. Med. 365, 1004, 2011), was a field trial of a vaccine based on the apical membrane region antigen 1 from $P$. falciparum in 400 Malian children. The study results suggest the vaccine might prevent only around one in five malaria infections from progressing to symptomatic disease, though there are signs that its efficacy might vary by strain.

Ashley Birkett, CSO of PATH MVI, says that MVI's refocusing of its portfolio at the end of the last decade is beginning to bear fruit with "very promising programs" that are progressing into the clinical stages. "Vaccines with higher levels of clinical efficacy than RTS,S, and also vaccines that block the cycle of transmission, really now form the basis of our portfolio," he says.
One possible candidate for higher efficacy is the liver-stage vaccine PfSPZ being developed by Rockville, Maryland-headquartered Sanaria, which delivers whole, live, attenuated, nonreplicating sporozoites harvested from the salivary glands of irradiated mosquitoes. In controlled trials, irradiated sporozoites delivered by mosquito bite to a small number of humans in a controlled setting induced levels of protective efficacy exceeding 90\% (J. Infect. Dis. 185, 1155, 2002). However, working out a vaccine administration method that doesn't involve a mosquito bite is a big challenge, says Stephen Hoffman, chief executive and scientific officer at Sanaria. An early-stage clinical trial on 80 humans published in September (Science 334, 475, 2011) showed the vaccine to be safe but the immune response and protection levels after subcutaneous administration were disappointingly low. "It wasn't the optimal way [of] administering it and we didn't get optimal responses, so we went back to the lab, to the nonhuman primate studies, figured out how to inoculate it and have now gone back into a clinical trial," says Hoffman.

The first data from the follow-up trial is expected in the fall next year, and a series of 11 trials over the next 12-15 months will investigate the optimal route of administration. "Our goal is to get a high level of protection, and we'll keep pushing until we get $80-90 \%$ protection for a long period of time," says Hoffman. "If we have that, then I think we can make enormous progress on the morbidity, mortality and transmission of malaria worldwide."

Simon Franz London

\title{
Interest groups jostle to influence PDUFA V
}

A finalized package of formal recommendations for the Prescription Drug User Fee Act $\mathrm{V}$ (PDUFA V) - the first came in 1992-is headed to US Congress this January. Its contents reflect many rounds of negotiations involving the US Food and Drug Administration (FDA), industry, medical groups, patient and consumer representatives and the general public.

"Please, stick with the main recommendations" seems to be the unofficial and nearuniversal plea - one that seemed to be shared among many of the major stakeholders who met late in October at the latest in a series of FDA-convened public sessions on PDUFA V. The looming question is, what will Congress do between January and September, when authority under PDUFA IV, formally known as the Food and Drug Administration Amendments Act of 2007 (FDAAA), expires?

With 2012 being a presidential election year, all kinds of things might happen. One

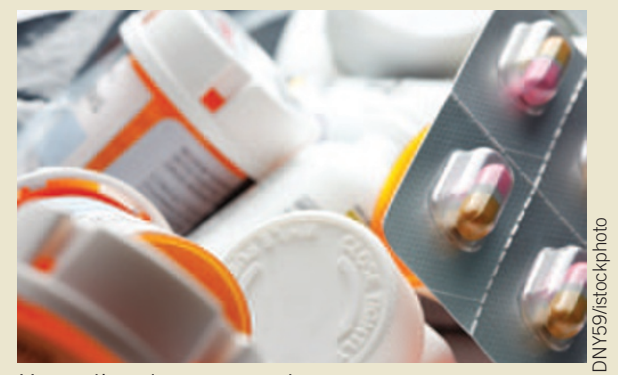

Upgrading drug approval. concern is that Congress will not meet its September deadline for reauthorizing PDUFA V. Such a mishap would stop the flow of resources from user fees and thus might force FDA to cut staff, a demoralizing and disruptive move even if short term. Another concern is that members of Congress might once again, as happened with FDAAA, expand FDA mandates, adding regulatory responsibilities without resources to support those activities.

"Even the threat of downsizing would be devastating to FDA," says Andrew Emmett, managing director of science and regulatory affairs at the Biotechnology Industry Organization (BIO) in Washington, DC, alluding to what might happen if Congress fails to meet the September reauthorization deadline. "BIO supports PDUFA V." In that same vein, BIO president Jim Greenwood lauds the PDUFA (and FDAAA) series, pointing to its aggregate success in helping to bring more than 1,200 new medicines to market since 1992 , many of them moving through regulatory reviews more rapidly than in the era before PDUFA.

The singular PDUFA innovation was to institute user fees. This extra revenue was directed to the FDA, allowing it to hire more professional staff. In turn, agency staff was subjected to tighter product review deadlines as well as higher performance standards, all directed at keeping reviews moving at a brisk pace. Since 2005, agency performance standards in terms of meeting review deadlines have hovered at $90 \%$ or higher, except for five quarters during 2008 and 2009, according to Theresa Mullin, director of the FDA Office of Planning and Informatics. Even when the agency missed deadlines, it often led to better discussions between companies and agency officials, sometimes accelerating overall product licensure times, she says.

Although many stakeholders embrace the concept of PDUFA V, they are far from unanimous about its details. Some disagreements epitomize a central FDA dilemma over product review stringency versus speed of reviews. For example, several organizations representing patients, including the Friends of Cancer Research, National Health Council and National Organization for Rare Disorders (NORD), claim FDA errs on the side of caution, not recognizing that many patients with chronic disorders or life-threatening cancers are 'risk tolerant' and apt to seek access to experimental treatments. Meanwhile, organizations such as the National Consumers League (NCL) say FDA is too focused on expediting drug approvals while neglecting its duties to protect consumers.

Additionally, several organizations raise concerns over conflicts of interest among outside experts who serve on FDA advisory panels. However, NORD vice president for public policy, Diane Dorman, says that the current conflict-of-interest provisions at FDA already suffer from being "out of balance," and thus too likely to disqualify experts who tend to be in short supply when called upon to review candidate products for treating patients with rare disorders. "We support eliminating provisions that apply only to FDA," she says.

Some consumer advocates rail against direct-to-consumer advertising, calling it misleading to the public. NCL executive director Sally Greenberg is calling for additional user fees to PDUFA V to support FDA reviews of such advertising materials before they are aired or published. 